\title{
Review Article \\ Elementary Superconductivity in Nonlinear Electrodynamics Coupled to Gravity
}

\author{
Irina Dymnikova ${ }^{1,2}$ \\ ${ }^{1}$ A.F. Ioffe Physico-Technical Institute, Politekhnicheskaja 26, Saint Petersburg 194021, Russia \\ ${ }^{2}$ Department of Mathematics and Computer Science, University of Warmia and Mazury, Słoneczna 54, 10-710 Olsztyn, Poland \\ Correspondence should be addressed to Irina Dymnikova; irina@uwm.edu.pl
}

Received 23 May 2015; Accepted 16 June 2015

Academic Editor: Cosimo Bambi

Copyright (C) 2015 Irina Dymnikova. This is an open access article distributed under the Creative Commons Attribution License, which permits unrestricted use, distribution, and reproduction in any medium, provided the original work is properly cited.

\begin{abstract}
Source-free equations of nonlinear electrodynamics minimally coupled to gravity admit regular axially symmetric asymptotically Kerr-Newman solutions which describe charged rotating black holes and electromagnetic spinning solitons (lumps). Asymptotic analysis of solutions shows, for both black holes and solitons, the existence of de Sitter vacuum interior which has the properties of a perfect conductor and ideal diamagnetic and displays superconducting behaviour which can be responsible for practically unlimited lifetime of the electron. Superconducting current flows on the equatorial ring replacing the Kerr ring singularity of the Kerr-Newman geometry. Interior de Sitter vacuum supplies the electron with the finite positive electromagnetic mass related to the interior de Sitter vacuum of the electroweak scale and to breaking of space-time symmetry, which allows explaining the mass-square differences for neutrino and the appearance of the minimal length scale in the annihilation reaction $e^{+} e^{-} \rightarrow \gamma \gamma(\gamma)$.
\end{abstract}

\section{Introduction}

Quantum electrodynamics applies the point-like idealization for leptons, which well describes in- and out-states of particles at the distances sufficiently large as compared with their eventual sizes. In experiments on high energy scattering, leptons are found structureless down to $\sim 10^{-16} \mathrm{~cm}$. There exist however experiments, in which particles approach each other so close that their annihilation results in their complete destruction. Study of the electromagnetic reaction $e^{+} e^{-} \rightarrow$ $\gamma \gamma(\gamma)$, with using the data from VENUS, TOPAZ, ALEPH, DELPHI, L3, and OPAL, reveals with the $5 \sigma$ significance the existence of the minimal length $l_{e}=1.57 \times 10^{-17} \mathrm{~cm}$ at the scale $E=1.253 \mathrm{TeV}$ [1]. The annihilation reaction can be a source of information about possible internal structure of leptons, which requires an extended model for the electron.

In 1962 Dirac proposed to assume the electron to have a finite size, with no a priori constraints fixing its size and shape [2]. In his model the electron is visualized as a spherical shell which serves as a source of electromagnetic field and is supplied with a cohesive force (Poincaré stress) of a nonelectromagnetic origin, needed to prevent the electron from flying apart under the Coulomb repulsion [2].
The Kerr-Newman solution to the source-free MaxwellEinstein equations found in 1965 [3]:

$$
\begin{aligned}
d s^{2}= & \frac{\left(2 m r-e^{2}\right)-\Sigma}{\Sigma} d t^{2}+\frac{\Sigma}{\Delta} d r^{2}+\Sigma d \theta^{2} \\
& -\frac{2 a\left(2 m r-e^{2}\right) \sin ^{2} \theta}{\Sigma} d t d \phi \\
& +\left(r^{2}+a^{2}+\frac{\left(2 m r-e^{2}\right) a^{2} \sin ^{2} \theta}{\Sigma}\right) \sin ^{2} \theta d \phi^{2} ; \\
\Delta & =r^{2}-2 m r+a^{2}+e^{2},
\end{aligned}
$$

where $\Sigma$-function and the associated electromagnetic potential read

$$
\begin{aligned}
\Sigma & =r^{2}+a^{2} \cos ^{2} \theta \\
A_{i} & =-\frac{e r}{\Sigma}\left[1 ; 0,0,-a \sin ^{2} \theta\right],
\end{aligned}
$$

inspired the further search since Carter discovered in 1968 that the parameter $a$ couples with the mass $m$ to give the 
angular momentum $J=m a$ and independently couples with the charge $e$ to give an asymptotic magnetic dipole moment $\mu=e a$, so that the gyromagnetic ratio $e / m$ is exactly the same as predicted for a spinning particle by the Dirac equation [4].

At the same time Carter discovered the big trouble of the Kerr-Newman geometry just in the case appropriate for the electron, $a^{2}+e^{2}>m^{2}$; when there are no Killing horizons, the manifold is geodesically complete (except for geodesics which reach the singularity), and any point can be connected to any other point by both a future and a past directed time-like curve. Closed time-like curves originate in the region where $g_{\phi \phi}<0$, can extend over the whole manifold and cannot be removed by taking a covering space [4].

The source models for the Kerr-Newman exterior fields, involving a screening or covering of causally dangerous region and Poincaré stress of different origins, can be roughly divided into disk-like [5-8], shell-like [9-12], bag-like [13-19], and string-like ([20] and references therein). The problem of matching the Kerr-Newman exterior to a rotating material source does not have a unique solution, since one is free to choose arbitrarily the boundary between the exterior and the interior [5] as well as an interior model.

The Dirac proposal to approach the electron without a priori constraints on its size and shape can be applied in the context of the Coleman lump (physical soliton) as a nonsingular, nondissipative solution of finite energy holding itself together by its own self-interaction [21]. An appropriate instrument to shed some light on the purely electromagnetic reaction of $e^{+} e^{-}$annihilation is nonlinear electrodynamics coupled to gravity (NED-GR) (NED theories appear as lowenergy effective limits in certain models of string/M-theories [22-24].).

Nonlinear electrodynamics was proposed by Born and Infeld as founded on two basic points: to consider electromagnetic field and particles within the frame of one physical entity which is electromagnetic field; to avoid letting physical quantities become infinite [25]. In their theory a total energy is finite and particles are considered as singularities of the field, but it is also possible to obtain the finite electron radius by introducing an upper limit on the electric field [25].

The Born-Infeld program can be realized in nonlinear electrodynamics minimally coupled to gravity. Sourcefree NED-GR equations admit regular causally safe axially symmetric asymptotically Kerr-Newman solutions which describe regular rotating charged black holes and electromagnetic spinning solitons (lumps) [26, 27].

For any gauge-invariant Lagrangian $\mathscr{L}(F)$, stress-energy tensor of electromagnetic field

$$
\kappa T_{v}^{\mu}=-2 \mathscr{L}_{F} F_{v \alpha} F^{\mu \alpha}+\frac{1}{2} \delta_{v}^{\mu} \mathscr{L} ; \quad \kappa=8 \pi G,
$$

where $F_{\mu \nu}=\partial_{\mu} A_{\nu}-\partial_{\nu} A_{\mu}$ (Greek indices run from 0 to 3 ) and $\mathscr{L}_{F}=d \mathscr{L} / d F$, in the spherically symmetric case, has the algebraic structure

$$
T_{0}^{0}=T_{1}^{1} \quad\left(p_{r}=-\rho\right)
$$

since the only essential components of $F_{\mu \nu}$ are a radial electric field $F_{01}$ and a radial magnetic field $F_{23}$. Regular spherically symmetric solutions with stress-energy tensors specified by (4) satisfying the weak energy condition (nonnegativity of density as measured by any local observe) have obligatory de Sitter center with $p=-\rho$ [28-31]. In NED-GR regular spherical solutions the weak energy condition is always satisfied and de Sitter vacuum provides a proper cut-off on self-interaction divergent for a point charge $[26,32]$. They can be transformed into regular axially symmetric solutions by the Gürses-Gürsey algorithm [33, 34].

Here we outline the generic properties of regular rotating charged black holes and solitons.

\section{Basic Equations}

Nonlinear electrodynamics minimally coupled to gravity is described by the action

$$
S=\frac{1}{16 \pi G} \int d^{4} x \sqrt{-g}[R-\mathscr{L}(F)] ; \quad F=F_{\mu \nu} F^{\mu \nu}
$$

where $R$ is the scalar curvature. The Lagrangian $\mathscr{L}(F)$ is an arbitrary function of $F$ which should have the Maxwell limit, $\mathscr{L} \rightarrow F, \mathscr{L}_{F} \rightarrow 1$ in the weak field regime.

Variation with respect to $A^{\mu}$ and $g_{\mu \nu}$ yields the dynamic field equations

$$
\begin{aligned}
\nabla_{\mu}\left(\mathscr{L}_{F} F^{\mu \nu}\right) & =0 ; \\
\nabla_{\mu}{ }^{*} F^{\mu \nu} & =0 ; \\
{ }^{*} F^{\mu \nu} & =\frac{1}{2} \eta^{\mu \nu \alpha \beta} F_{\alpha \beta} ; \\
\eta^{0123} & =-\frac{1}{\sqrt{-g}}
\end{aligned}
$$

and the Einstein equation $G_{\mu \nu}=-\kappa T_{\mu \nu}$ with $T_{\mu \nu}$ given by (3).

NED-GR equations do not admit regular spherically symmetric solutions with the Maxwell center [35], but they admit regular solutions with the de Sitter center [32]. The question of correct description of NED-GR regular electrically charged structures by the Lagrange dynamics is clarified in [36]. Regular solutions satisfying (4) are described by the metric

$$
\begin{aligned}
& d s^{2}=g(r) d t^{2}-\frac{d r^{2}}{g(r)}-r^{2} d \Omega^{2} ; \\
& \quad g(r)=1-\frac{2 \mathscr{M}(r)}{r} ; \mathscr{M}(r)=4 \pi \int_{0}^{r} \tilde{\rho}(x) x^{2} d x,
\end{aligned}
$$

with the electromagnetic density $\widetilde{\rho}(r)=T_{t}^{t}(r)$ from (3). This metric is asymptotically de Sitter as $r \rightarrow 0$, and asymptotically Reissner-Nordström as $r \rightarrow \infty$ [32].

The regular spherical solutions generated by (4) belong to the Kerr-Schild class $[18,37,38]$ and can be transformed by the Gürses-Gürsey algorithm [33] into regular axially symmetric solutions which describe regular rotating electrically charged objects, asymptotically Kerr-Newman for a distant observer $[26,34]$. 
In the Boyer-Lindquist coordinates the rotating metric reads (in the units $G=c=1$ ) [33]

$$
\begin{aligned}
d s^{2}= & \frac{2 f(r)-\Sigma}{\Sigma} d t^{2}+\frac{\Sigma}{\Delta} d r^{2}+\Sigma d \theta^{2} \\
& -\frac{4 a f(r) \sin ^{2} \theta}{\Sigma} d t d \phi \\
& +\left(r^{2}+a^{2}+\frac{2 f(r) a^{2} \sin ^{2} \theta}{\Sigma}\right) \sin ^{2} \theta d \phi^{2},
\end{aligned}
$$

where $\Delta=r^{2}+a^{2}-2 f(r)$. A function $f(r)=r \mathscr{M}(r)$ comes from a spherically symmetric solution [33]. For the Kerr-Newman geometry $2 f(r)=2 m r-e^{2}$ is responsible for causality violation related to regions where $g_{\phi \phi}<0$ in (1). For NED-GR regular solutions satisfying the weak energy condition, $\mathscr{M}(r)$ is nonnegative function monotonically growing from $4 \pi \tilde{\rho}(0) r^{3} / 3$ as $r \rightarrow 0$ to $m-e^{2} / 2 r$ as $r \rightarrow \infty$ [32]. This guarantees the causal safety on the whole manifold due to $f(r) \geq 0$ and $g_{\phi \phi}>0$ in (8).

The coordinate $r$ is defined as an affine parameter along either of two principal null congruences, and the surfaces of constant $r$ are the oblate confocal ellipsoids of revolution

$$
r^{4}-r^{2}\left(x^{2}+y^{2}+z^{2}-a^{2}\right)-a^{2} z^{2}=0,
$$

which degenerate, for $r=0$, to the equatorial disk

$$
\begin{aligned}
x^{2}+y^{2} & \leq a^{2}, \\
z & =0,
\end{aligned}
$$

centered on the symmetry axis and bounded by the ring $x^{2}+$ $y^{2}=a^{2}(r=0, \theta=\pi / 2)$ [39].

\section{Geometry}

The Cartesian coordinates $x, y$, and $z$ are related to the BoyerLindquist coordinates $r, \theta$, and $\phi$ by

$$
\begin{aligned}
x^{2}+y^{2} & =\left(r^{2}+a^{2}\right) \sin ^{2} \theta ; \\
z & =r \cos \theta .
\end{aligned}
$$

The anisotropic stress-energy tensor responsible for (8) can be written in the form [33]

$$
T_{\mu \nu}=\left(\rho+p_{\perp}\right)\left(u_{\mu} u_{\nu}-l_{\mu} l_{\nu}\right)+p_{\perp} g_{\mu \nu}
$$

in the orthonormal tetrad

$$
\begin{aligned}
u^{\mu} & =\frac{1}{\sqrt{ \pm \Delta \Sigma}}\left[\left(r^{2}+a^{2}\right) \delta_{0}^{\mu}+a \delta_{3}^{\mu}\right], \\
l^{\mu} & =\sqrt{\frac{ \pm \Delta}{\Sigma}} \delta_{1}^{\mu}, \\
n^{\mu} & =\frac{1}{\sqrt{\Sigma}} \delta_{2}^{\mu}, \\
m^{\mu} & =\frac{-1}{\sqrt{\Sigma} \sin \theta}\left[a \sin ^{2} \theta \delta_{0}^{\mu}+\delta_{3}^{\mu}\right] .
\end{aligned}
$$

The sign plus refers to the regions outside the event horizon and inside the Cauchy horizon where vector $u^{\mu}$ is time-like, and the sign minus refers to the regions between the horizons where vector $l^{\mu}$ is time-like. Vectors $m^{\mu}$ and $n^{\mu}$ are space-like in all regions.

The eigenvalues of the stress-energy tensor (3) in the corotating frame where each of ellipsoidal layers rotates with the angular velocity $\omega(r)=u^{\phi} / u^{t}=a /\left(r^{2}+a^{2}\right)$ [18] are defined by

$$
\begin{aligned}
T_{\mu \nu} u^{\mu} u^{\nu} & =\rho(r, \theta) ; \\
T_{\mu \nu} l^{\mu} l^{\nu} & =p_{r}=-\rho ; \\
T_{\mu \nu} n^{\mu} n^{\nu} & =T_{\mu \nu} m^{\mu} m^{\nu}=p_{\perp}(r, \theta),
\end{aligned}
$$

in the regions outside the event horizon and inside the Cauchy horizon where density is defined as the eigenvalue of the time-like eigenvector $u^{\mu}$. They are related to function $f(r)$ [18] as $\kappa \Sigma^{2} \rho=2\left(f^{\prime} r-f\right) ; \kappa \Sigma^{2} p_{\perp}=2\left(f^{\prime} r-f\right)-f^{\prime \prime} \Sigma[18]$. This gives

$$
\begin{aligned}
\kappa \rho(r, \theta) & =\frac{r^{4}}{\Sigma^{2}} \tilde{\rho}(r) ; \\
\kappa\left(p_{\perp}+\rho\right) & =2\left(\frac{r^{4}}{\Sigma^{2}}-\frac{r^{2}}{\Sigma}\right) \tilde{\rho}(r)-\frac{r^{3}}{2 \Sigma} \tilde{\rho}^{\prime}(r) \\
& =\frac{2 r^{2}}{\Sigma^{2}}\left(\frac{\Sigma r}{4}\left|\tilde{\rho}^{\prime}\right|-\tilde{\rho} a^{2} \cos ^{2} \theta\right),
\end{aligned}
$$

where $\widetilde{\rho}(r)$ is a relevant spherically symmetric density profile. The prime denotes the derivative with respect to $r$.

3.1. Horizons, Ergospheres, and Ergoregions. Horizons are defined by zeros of function $\Delta(r)$ given by

$$
\Delta(r)=r^{2}+a^{2}-2 f(r)=a^{2}+r^{2} g(r) .
$$

$\Delta=a^{2}$ at zero points of the metric function $g\left(r_{h}\right)=0$ and changes from $\Delta=a^{2}$ as $r=0$ to $\Delta \rightarrow \infty$ as $r \rightarrow \infty$.

Ergosphere is a surface of a static limit $g_{t t}=0$ given by

$$
g_{t t}(r, \theta)=r^{2}+a^{2} \cos ^{2} \theta-2 f(r)=0 .
$$

It follows that $z^{2}=\left(2 r^{2} f(r)-r^{4}\right) / a^{2}$. Each point of the ergosphere belongs to some of confocal ellipsoids (9) covering the whole space as the coordinate surfaces $r=$ const. At the $z$-axis (16) and (17) are identical, so that the minor axis of ergosphere is equal to $r_{+}$.

For black holes ergoregions (the regions where $g_{t t}<$ 0 ) exist for any density profile. Black holes have at most two horizons. Ergoregions exist between the event horizon and ergosphere. Solitons are objects without horizons; they can have two, one, or no ergospheres, this depends on the particular form of a density profile $\widetilde{\rho}(r)$ and on the values of parameters [27].

3.2. De Sitter Vacuum Interiors. Rotation transforms the de Sitter center to the de Sitter equatorial disk (10) which exists 
in each regular axially symmetric geometry. In the limit $r \rightarrow$ 0 , on disk (10), $r^{2} / \Sigma \rightarrow 1$ [26]. For the spherical solutions regularity requires $\tilde{\rho}(r)<\infty, r \tilde{\rho}^{\prime}(r) \rightarrow 0$, and $2 f(r) \rightarrow$ $\kappa \widetilde{\rho}(0) r^{4} / 3$ as $r \rightarrow 0$ [32], so that disk $r=0$ is intrinsically flat [26]. Equation (15) gives in this limit the equation of state on the disk

$$
p_{\perp}+\rho=0 \longrightarrow p_{\perp}=p_{r}=-\rho,
$$

which represents the rotating de Sitter vacuum [26].

Equation (15) implies a possibility of generic violation of the weak energy condition (WEC) which was reported for several particular models of regular rotating objects [18, $40-42]$. WEC can be violated beyond the vacuum surface $\mathscr{E}(r, z)=0$ on which $p_{\perp}+\rho=0$ and the right-hand side in (15) can change its sign [27]. It can be expressed through the pressure of a related spherical solution, $\overline{p_{\perp}}=-\tilde{\rho}-r \tilde{\rho}^{\prime} / 2$ [32], which gives [27]

$$
\begin{aligned}
\kappa\left(p_{\perp}+\rho\right) & =\frac{r\left|\tilde{\rho}^{\prime}\right|}{2 \Sigma^{2}} \mathscr{E}(r, z)=0 ; \\
\mathscr{E}(r, z) & =\left(r^{4}-z^{2} P(r)\right) ; P(r)=\frac{2 a^{2}}{r\left|\widetilde{\rho}^{\prime}\right|}\left(\widetilde{\rho}-\widetilde{p_{\perp}}\right) .
\end{aligned}
$$

The existence of vacuum surfaces is directly stipulated by fulfillment of the dominant energy condition $\left(\widetilde{\rho} \geq \widetilde{p_{k}}\right)$ for related spherical solutions. Each vacuum $\mathscr{E}$-surface contains the de Sitter disk as a bridge and is entirely confined within the $r_{*}$-ellipsoid whose minor axis coincides with $|z|_{\max }$ for the $\mathscr{E}$-surface [27]. The squared width of the $\mathscr{E}$-surface $W_{\mathscr{E}}^{2}=$ $\left(x^{2}+y^{2}\right)_{\mathscr{E}}=\left(a^{2}+|z| \sqrt{P(r)}\right)(1-|z| / \sqrt{P(r)})$. For regular solutions $r \tilde{\rho}^{\prime} \rightarrow 0, p_{\perp} \rightarrow-\rho$ as $r \rightarrow 0$ [32], and $P(r) \rightarrow A^{2} r^{-(n+1)}$ with the integer $n \geq 0$ as $r \rightarrow 0$. Function $W_{\mathscr{E}}(z)$ has the cusp at approaching the disk and at least two symmetric maxima between $z= \pm r_{*}$ and $z=0$ [27].

In Figure 1 [27] $\mathscr{E}$-surface is plotted for the electromagnetic soliton with the regularized Coulomb profile [32]:

$$
\tilde{\rho}=\frac{q^{2}}{\left(r^{2}+r_{q}^{2}\right)^{2}} ; \quad r_{q}=\frac{\pi q^{2}}{8 m} .
$$

Its width in the equatorial plane $W_{\mathscr{E}}=a$ and the height $H_{\mathscr{C}}=$ $|z|_{\max }=\sqrt{a r_{q}}$. For the electron $W_{\mathscr{E}}=\lambda_{e} / 2$, where $\lambda_{e} \simeq 3.9 \times$ $10^{-11} \mathrm{~cm}, H_{\mathscr{E}} \simeq 0.038 \lambda_{e}$ and $\eta=W_{\mathscr{E}} / H_{\mathscr{C}}=\sqrt{a / r_{q}} \simeq 13.2$.

\section{Electromagnetic Fields}

Nonzero field components compatible with the axial symmetry are $F_{01}, F_{02}, F_{13}$, and $F_{23}$. In geometry with the metric (8) they are related by

$$
\begin{aligned}
F_{31} & =a \sin ^{2} \theta F_{10} ; \\
a F_{23} & =\left(r^{2}+a^{2}\right) F_{02} .
\end{aligned}
$$

The field invariant $F=F_{\mu \nu} F^{\mu \nu}$ in the axially symmetric case reduces to

$$
F=2\left(\frac{F_{20}^{2}}{a^{2} \sin ^{2} \theta}-F_{10}^{2}\right) .
$$

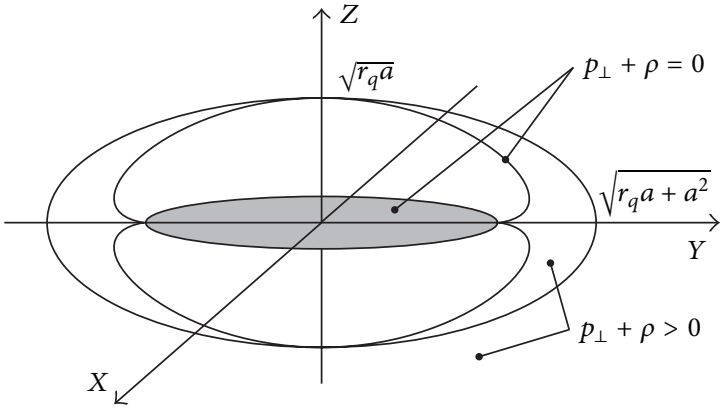

FIGURE 1: Electromagnetic $e$-lump from nonlinear electrodynamics coupled to gravity [27].

In terms of the 3 vectors, denoted by Latin indices running from 1 to 3 and defined as

$$
\begin{aligned}
E_{j} & =\left\{F_{j 0}\right\} ; \\
D^{j} & =\left\{\mathscr{L}_{F} F^{0 j}\right\} ; \\
B^{j} & =\left\{{ }^{*} F^{j 0}\right\} ; \\
H_{j} & =\left\{\mathscr{L}_{F}{ }^{*} F_{0 j}\right\},
\end{aligned}
$$

the field equations (6) take the form of the Maxwell equations. The electric induction $D$ and the magnetic induction $B$ are related with the electric and magnetic field intensities by [26]

$$
\begin{aligned}
& D^{j}=\epsilon_{k}^{j} E^{k} ; \\
& B^{j}=\mu_{k}^{j} H^{k},
\end{aligned}
$$

where $\epsilon_{j}^{k}$ and $\mu_{j}^{k}$ are the tensors of the electric and magnetic permeability given by [26]

$$
\begin{aligned}
\epsilon_{r}^{r} & =\frac{\left(r^{2}+a^{2}\right)}{\Delta} \mathscr{L}_{F} ; \\
\epsilon_{\theta}^{\theta} & =\mathscr{L}_{F} ; \\
\mu_{r}^{r} & =\frac{\left(r^{2}+a^{2}\right)}{\Delta \mathscr{L}_{F}} ; \\
\mu_{\theta}^{\theta} & =\frac{1}{\mathscr{L}_{F}} .
\end{aligned}
$$

The dynamical equations (6) are satisfied by the functions [26]

$$
\begin{aligned}
F_{10} & =\frac{q}{\sum^{2} \mathscr{L}_{F}}\left(r^{2}-a^{2} \cos ^{2} \theta\right) ; \\
F_{02} & =\frac{q}{\sum^{2} \mathscr{L}_{F}} a^{2} r \sin 2 \theta ; \\
F_{31} & =a \sin ^{2} \theta F_{10} ; \\
a F_{23} & =\left(r^{2}+a^{2}\right) F_{02}
\end{aligned}
$$


in the weak field limit $\mathscr{L}_{F}=1$, where they coincide with the Kerr-Newman fields $[4,16]$, and an integration constant $q$ is identified as the electric charge. For the electron $q=-e$, $m a=\hbar / 2$ [4], $a=\lambda_{e} / 2$, where $\lambda_{e}=\hbar /\left(m_{e} c\right)$ is the Compton wavelength. In the observer region $r \gg \lambda_{e}$,

$$
\begin{aligned}
& E_{r}=-\frac{e}{r^{2}}\left(1-\frac{\hbar^{2}}{m_{e}^{2} c^{2}} \frac{3 \cos ^{2} \theta}{4 r^{2}}\right) ; \\
& E_{\theta}=\frac{e \hbar^{2}}{m_{e}^{2} c^{2}} \frac{\sin 2 \theta}{4 r^{3}} ; \\
& B^{r}=-\frac{e \hbar}{m_{e} c} \frac{\cos \theta}{r^{3}}=2 \mu_{e} \frac{\cos \theta}{r^{3}} ; \\
& B_{\theta}=-\mu_{e} \frac{\sin \theta}{r^{4}} .
\end{aligned}
$$

The Planck constant appears here due to ability, discovered by Carter, of the Kerr-Newman solution to present the electron as seen by a distant observer. In terms of the Coleman lump (27) describes the following situation: the leading term in $E_{r}$ gives the Coulomb law as the classical limit $\hbar=0$; the higher terms represent the quantum corrections.

With taking into account connection between the field components (21), we have four dynamical equations (6) for two field components, $F_{01}, F_{02}$, and the nonlinearity function $\mathscr{L}_{F}$. Condition of compatibility of system of four equations for three function reduces to the constraint on the nonlinearity which has the form [27]

$$
\begin{aligned}
\frac{\partial}{\partial r}( & \left.\frac{1}{L_{F}} \frac{\partial L_{F}}{\partial \theta}\right) \frac{\partial}{\partial \theta}\left(\frac{1}{L_{F}} \frac{\partial L_{F}}{\partial r}\right) \\
& +\frac{4 a^{2} \sin ^{2}(\theta)}{\Sigma^{2}} \frac{1}{L_{F}^{2}}\left[r \frac{\partial L_{F}}{\partial r}+\cot (\theta) \frac{\partial L_{F}}{\partial \theta}\right]^{2}=0 .
\end{aligned}
$$

The functions (26) present asymptotic solutions to the dynamical equations (6) in the limit $\mathscr{L}_{F} \rightarrow \infty$ and $\mathscr{L}_{F} \Sigma^{2} \rightarrow$ $\infty$. In this limit they satisfy the system (6) and the condition of their compatibility (28) [27].

\section{Interior Dynamics and Elementary Superconductivity}

The relation connecting density and pressure with the electromagnetic fields reads [26]

$$
\kappa\left(p_{\perp}+\rho\right)=2 \mathscr{L}_{F}\left(F_{10}^{2}+\frac{F_{20}^{2}}{a^{2} \sin ^{2} \theta}\right) .
$$

In the limit $\mathscr{L}_{F} \rightarrow \infty$, (6) have asymptotic solutions (26) $[26,27]$. It results in [26]

$$
\kappa\left(p_{\perp}+\rho\right)=\frac{2 q^{2}}{\mathscr{L}_{F} \Sigma^{2}} .
$$

Equation of state on the disk (18) dictated by geometry for regular spinning solutions requires $p_{\perp}+\rho=0$. It follows that
$\mathscr{L}_{F} \Sigma^{2} \rightarrow \infty$ and hence $\mathscr{L}_{F} \rightarrow \infty$, since $\Sigma \rightarrow 0$ on the disk.

The magnetic induction $B$ vanishes in this limit, so that $B \rightarrow 0$ on the disk [26]. The electric permeability in (25) goes to infinity; the magnetic permeability $\mu=\mathscr{L}_{F}{ }^{-1}$ vanishes, so that the de Sitter vacuum disk has both perfect conductor and ideal diamagnetic properties.

In electrodynamics of continued media the transition to a superconducting state corresponds to the limits $B \rightarrow 0$ and $\mu \rightarrow 0$ in a surface current $j_{s}=((1-\mu) / 4 \pi \mu)[n B]$ where $n$ is the normal to the surface; the right-hand side then becomes indeterminate, and there is no condition which would restrict the possible values of the current [43]. Definition of a surface current for a charged surface layer is $4 \pi j_{k}=\left[e_{(k)}^{\alpha} F_{\alpha \beta} n^{\beta}\right][5]$, where [.] denotes a jump across the layer; $e_{(k)}^{\alpha}$ are the tangential base vectors associated with the intrinsic coordinates on the disk $t, \phi, 0 \leq \xi \leq \pi / 2 ; n_{\alpha}=$ $\left(1+q^{2} / a^{2}\right)^{-1 / 2} \cos \xi \delta_{\alpha}^{1}$ is the unit normal directed upwards [5]. With using asymptotic solutions (26) and magnetic permeability $\mu=1 / \mathscr{L}_{F}$, we obtain the surface current [44]

$$
j_{\phi}=-\frac{q}{2 \pi a} \sqrt{1+\frac{q^{2}}{a^{2}} \sin ^{2} \xi \frac{\mu}{\cos ^{3} \xi} .}
$$

At approaching the ring $r=0, \xi=\pi / 2$, both terms in the second fraction go to zero quite independently. As a result the surface currents on the ring can be any and amount to a nonzero total value $[26,44]$.

The superconducting current (31) replaces the Kerr ring singularity of the Kerr-Newman geometry and can be considered as a source of the Kerr-Newman fields. This kind of a source is nondissipative, so that electrovacuum solitons present actually e-lumps in accordance with the Coleman definition of the lump as a nonsingular, nondissipative solution of finite energy holding itself together by its own selfinteraction [21]. Lifetime of $e$-lump is unlimited.

De Sitter disk exists in the interior of any regular charged black hole and soliton. When a related spherical solution satisfies the dominant energy condition, it exists as a bridge inside the $\mathscr{E}$-surface, defined by $p_{\perp}+\rho=0$. It follows that $\mathscr{L}_{F} \rightarrow \infty$ by virtue of (30). The magnetic permeability vanishes and electric permeability goes to infinity, so that $\mathscr{E}$ surface displays the properties of a perfect conductor and ideal diamagnetic. Also magnetic induction vanishes on $\mathscr{E}$ surface by virtue of the asymptotic solutions (26), so that the Meissner effect occurs there [26]. Within $\mathscr{E}$-surface, in cavities between its upper and down boundaries and the bridge, negative value of $\left(p_{\perp}+\rho\right)$ in (29) would mean negative values for electric and magnetic permeabilities inadmissible in electrodynamics of continued media [43]. The case, favored by the underlying idea of nonlinearity replacing a singularity and suggested by vanishing of magnetic induction on the surrounding $\mathscr{E}$-surface, is extension of $\mathscr{L}_{F} \rightarrow \infty$ to its interiors. Then we have de Sitter vacuum core, $p=$ $-\rho$, with the properties of a perfect conductor and ideal diamagnetic and magnetic fields vanishing throughout the whole core, and the weak energy condition is satisfied for regular rotating charged black holes and solitons. 


\section{Summary and Discussion}

Nonlinear electrodynamics minimally coupled to gravity admits the regular axially symmetric solutions asymptotically Kerr-Newman for a distant observer, which describe regular charged rotating black holes and electromagnetic solitons.

The basic generic feature of all these objects is the interior de Sitter vacuum disk with $p=-\rho$ in the corotating frame, which has properties of a perfect conductor and ideal diamagnetic. Superconducting current flows on the equatorial ring replacing the Kerr singularity of the Kerr-Newman geometry. This current serves as a nondissipative source of the KerrNewman external fields and can be responsible for practically unlimited lifetime of the electron.

In the case when related spherical solution satisfies the dominant energy condition, de Sitter disk is incorporated as a bridge in the vacuum $\mathscr{E}$-surface with the equation of state $p=-\rho$, properties of the perfect conductor and ideal diamagnetic, and vanishing magnetic induction. This allows extending these properties to the interior of the $\mathscr{E}$-surface, since otherwise the violation of the weak energy condition in its interior would lead to the negative values of the electric and magnetic permeabilities, inadmissible in electrodynamics of continued media. As a result the weak energy condition is satisfied for regular rotating objects of this type.

Mass parameter $m$ in a NED-GR spinning solution is the electromagnetic mass $[26,32]$, related to interior de Sitter vacuum and breaking of space-time symmetry from the de Sitter group for any solution specified by (4) [30]. This has been tested by application of Casimir invariants of the de Sitter group in the region surrounding the interaction vertex for the sub-eV particles, which predicts $\mathrm{TeV}$ scale for gravitoelectroweak unification and explains the experimental results known as a negative mass-squared difference for neutrino [45].

This conforms with the Higgs mechanism for generation of mass via spontaneous breaking of symmetry of a scalar field vacuum. The Higgs field is involved in mass generation in its false vacuum state satisfying $p=-\rho$. Then the space-time symmetry around the interaction vertex is the de Sitter group, while in the observer region it is Poincaré group (strictly speaking another de Sitter with much less value of the cosmic vacuum density), which requires breaking of symmetry in between to the Lorentz radial boosts only [30]. Generation of mass by the Higgs mechanism must thus involve breaking of space-time symmetry [44].

Interior de Sitter vacuum can explain the appearance of the minimal length in the reaction $e^{+} e^{-} \rightarrow \gamma \gamma(\gamma)$. The definite feature of annihilation process is that at its certain stage a region of interaction is neutral and spinless. We can roughly model it by a spherical bag with de Sitter vacuum interior asymptotically Schwarzschild as $r \rightarrow \infty$. For such a structure there exists a zero gravity surface at which the strong energy condition $\left(\rho+\sum_{k} \geq 0\right)$ is violated and beyond which gravity becomes repulsive $[29,46]$. The related length scale $r_{s} \simeq\left(r_{0}^{2} r_{g}\right)^{1 / 3}$ appears in any geometry with de Sitter interior and Schwarzschild exterior [28, 47]. Adopting for de Sitter interior the vacuum expectation value $v=246 \mathrm{GeV}$ responsible for the electron mass [48], we get de Sitter radius $r_{0}=1.37 \mathrm{~cm}$. Radius $r_{s}$ at the energy scale $E \simeq 1.253 \mathrm{TeV}$ is $r_{s} \simeq 0.86 \times 10^{-16} \mathrm{~cm}$, so that the scale $l_{e}=1.57 \times 10^{-17} \mathrm{~cm}$ fits inside a region where gravity is repulsive. Regular NED-GR solutions provide a de Sitter cutoff on electromagnetic selfenergy, which can be qualitatively estimated by $e^{2} / r_{c}^{4} \simeq \kappa \rho_{0}=$ $3 / r_{0}^{2}$. It gives the length scale $r_{c}$ at which electromagnetic attraction is balanced by de Sitter gravitational repulsion $r_{c} \simeq$ $1.05 \times 10^{-17} \mathrm{~cm}$, sufficiently close to the experimental value $l_{e}$ for such a rough estimate [1]. The minimal length scale $l_{e}$ can be thus understood as a distance of the closest approach of annihilating particles at which electromagnetic attraction is stopped by the gravitational repulsion due to interior de Sitter vacuum.

\section{Conflict of Interests}

The author declares that there is no conflict of interests regarding the publication of this paper.

\section{References}

[1] I. Dymnikova, A. Sakharov, and J. Ulbricht, "Appearance of a minimal length in $e^{+} e^{-}$annihilation," Advances in High Energy Physics, vol. 2014, Article ID 707812, 9 pages, 2014.

[2] P. A. M. Dirac, "An extensible model of the electron," Proceedings of the Royal Society of London A, vol. 268, no. 1332, p. 57, 1962.

[3] E. T. Newman, E. Couch, K. Chinnapared, A. Exton, A. Prakash, and R. Torrence, "Metric of a rotating, charged mass," Journal of Mathematical Physics, vol. 6, no. 6, pp. 918-919, 1965.

[4] B. Carter, "Global structure of the Kerr family of gravitational fields," Physical Review, vol. 174, no. 5, pp. 1559-1571, 1968.

[5] W. Israel, "Source of the Kerr metric", Physical Review. D. Particles and Fields. Third Series, vol. 2, article 641, 1970.

[6] A. Y. Burinskii, "Microgeons with spin," Soviet Physics-JETP, vol. 39, p. 193, 1974.

[7] V. H. Hamuty, "An 'interior' of the Kerr metric," Physics Letters $A$, vol. 56, no. 2, pp. 77-78, 1976.

[8] C. A. López, "Material and electromagnetic sources of the KerrNewman geometry," Il Nuovo Cimento B, vol. 76, no. 1, pp. 9-27, 1983.

[9] V. de la Cruz, J. E. Chase, and W. Israel, "Gravitational collapse with asymmetries," Physical Review Letters, vol. 24, article 423, 1970.

[10] J. M. Cohen, "Note on the Kerr metric and rotating masses," Journal of Mathematical Physics, vol. 8, no. 7, p. 1477, 1967.

[11] R. H. Boyer, "Quantum electromagnetic zero-point energy of a conducting spherical shell and the Casimir model for a charged particle," Physical Review, vol. 174, no. 5, pp. 1764-1776, 1968.

[12] C. A. López, "Extended model of the electron in general relativity," Physical Review D, vol. 30, no. 2, p. 313, 1984.

[13] R. H. Boyer, "Rotating fluid masses in general relativity," Mathematical Proceedings of the Cambridge Philosophical Society, vol. 61, no. 2, pp. 527-530, 1965.

[14] R. H. Boyer, "Rotating fluid masses in general relativity. II," Mathematical Proceedings of the Cambridge Philosophical Society, vol. 62, no. 3, pp. 495-501, 1966.

[15] M. Trümper, "Einsteinsche Feldgleichungen für das axialsymmetrische, stationäre Gravitationsfeld im Innern einer starr 
rotierenden idealen Flüssigkeit," Zeitschrift für Naturforschung, vol. 22, pp. 1347-1351, 1967.

[16] J. Tiomno, "Electromagnetic field of rotating charged bodies," Physical Review D, vol. 7, article 992, 1973.

[17] A. Y. Burinskii, "The problem of the source of the KerrNewman metric: the volume Casimir effect and superdense pseudovacuum state," Physics Letters. B, vol. 216, no. 1-2, pp. 123$126,1989$.

[18] A. Burinskii, E. Elizalde, S. R. Hildebrandt, and G. Magli, "Regular sources of the Kerr-Schild class for rotating and nonrotating black hole solutions," Physical Review D, vol. 65, no. 6, Article ID 064039, 15 pages, 2002.

[19] A. Burinskii, "Kerr-Newman electron as spinning soliton," International Journal of Modern Physics A, vol. 29, no. 26, Article ID 1450133, 2014.

[20] A. Y. Burinskii, "Stringlike structures in Kerr-Schild geometry: the $N=2$ string, twistors, and the Calabi-Yau twofold," Theoretical and Mathematical Physics, vol. 177, no. 2, pp. 1492-1504, 2013.

[21] S. Coleman, "Classical lumps and their quantum descendants," in New Phenomena in Subnuclear Physics, A. Zichichi, Ed., pp. 297-421, Plenum, New York, NY, USA, 1977.

[22] E. S. Fradkin and A. A. Tseytlin, "Non-linear electrodynamics from quantized strings," Physics Letters B, vol. 163, no. 1-4, pp. 123-130, 1985.

[23] A. A. Tseytlin, "Vector field effective action in the open superstring theory," Nuclear Physics B, vol. 276, no. 2, pp. 391$428,1986$.

[24] N. Seiberg and E. Witten, "The D1/D5 system and singular CFT," Journal of High Energy Physics, vol. 1999, no. 4, article 032, 1999.

[25] M. Born and L. Infeld, "Foundations of the new field theory," Proceedings of the Royal Society of London A, vol. 144, no. 852, p. 425, 1934.

[26] I. Dymnikova, "Spinning superconducting electrovacuum soliton," Physics Letters B, vol. 639, no. 3-4, pp. 368-372, 2006.

[27] I. Dymnikova and E. Galaktionov, "Regular rotating electrically charged black holes and solitons in nonlinear electrodynamics minimally coupled to gravity," to appear in Classical and Quantum Gravity.

[28] I. Dymnikova, "Vacuum nonsingular black hole," General Relativity and Gravitation, vol. 24, no. 3, pp. 235-242, 1992.

[29] I. G. Dymnikova, “The algebraic structure of a cosmological term in spherically symmetric solutions," Physics Letters B, vol. 472, no. 1-2, pp. 33-38, 2000.

[30] I. Dymnikova, "The cosmological term as a source of mass," Classical and Quantum Gravity, vol. 19, no. 4, pp. 725-739, 2002.

[31] I. Dymnikova, "Spherically symmetric space-time with regular de sitter center," International Journal of Modern Physics D, vol. 12, no. 6, pp. 1015-1034, 2003.

[32] I. Dymnikova, "Regular electrically charged vacuum structures with de Sitter centre in nonlinear electrodynamics coupled to general relativity," Classical and Quantum Gravity, vol. 21, no. 18, pp. 4417-4428, 2004.

[33] M. Gürses and F. Gürsey, "Lorentz covariant treatment of the Kerr-Schild geometry," Journal of Mathematical Physics, vol. 16, no. 12, pp. 2385-2390, 1975.

[34] A. Burinskii and S. R. Hildebrandt, "New type of regular black holes and particlelike solutions from nonlinear electrodynamics," Physical Review D, vol. 65, no. 10, Article ID 104017, 2002.
[35] K. A. Bronnikov, "Regular magnetic black holes and monopoles from nonlinear electrodynamics," Physical Review D, vol. 63, no. 4, Article ID 044005, 6 pages, 2001.

[36] I. Dymnikova, E. Galaktionov, and E. Tropp, "Existence of electrically charged structures with regular center in nonlinear electrodynamics minimally coupled to gravity," Advances in Mathematical Physics, vol. 2015, Article ID 496475, 9 pages, 2015.

[37] R. P. Kerr and A. Schild, "Some algebraically degenerate solutions of Einstein's gravitational field equations," in Proceedings of the Symposia in Applied Mathematics, vol. 17, pp. 199-209, 1965.

[38] E. Elizalde and S. R. Hildebrandt, "Family of regular interiors for nonrotating black holes with $T_{0}^{0}=T_{1}^{1}$," Physical Review D, vol. 65, Article ID 124024, 2002.

[39] S. Chandrasekhar, The Mathematical Theory of Black Holes, The Clarendon Press, New York, NY, USA, 1983.

[40] J. C. S. Neves and A. Saa, "Regular rotating black holes and the weak energy condition," Physics Letters B, vol. 734, pp. 44-48, 2014.

[41] C. Bambi and L. Modesto, "Rotating regular black holes," Physics Letters B, vol. 721, no. 4-5, pp. 329-334, 2013.

[42] B. Toshmatov, B. Ahmedov, A. Abdujabbarov, and Z. Stuchlík, "Rotating regular black hole solution," Physical Review D Particles, Fields, Gravitation and Cosmology, vol. 89, no. 10, Article ID 104017, 2014.

[43] L. D. Landau and E. M. Lifshitz, Electrodynamics of Continued Media, Pergamon Press, 1993.

[44] I. Dymnikova, "Electromagnetic source for the Kerr-Newman geometry," International Journal of Modern Physics D. Submitted.

[45] D. V. Ahluwalia and I. Dymnikova, "A theoretical case for negative mass-square for sub-eV particles," International Journal of Modern Physics D, vol. 12, p. 1878, 2003.

[46] I. Dymnikova, "De Sitter-Schwarzschild black hole: its particlelike core and thermodynamical properties," International Journal of Modern Physics D, vol. 5, no. 5, pp. 529-540, 1996.

[47] E. Poisson and W. Israel, "Structure of the black hole nucleus," Classical and Quantum Gravity, vol. 5, no. 12, pp. L201-L205, 1988.

[48] C. Quigg, Gauge Theories of the Strong, Weak, and Electromagnetic Interactions, Addison-Wesley Publishing, 1983. 

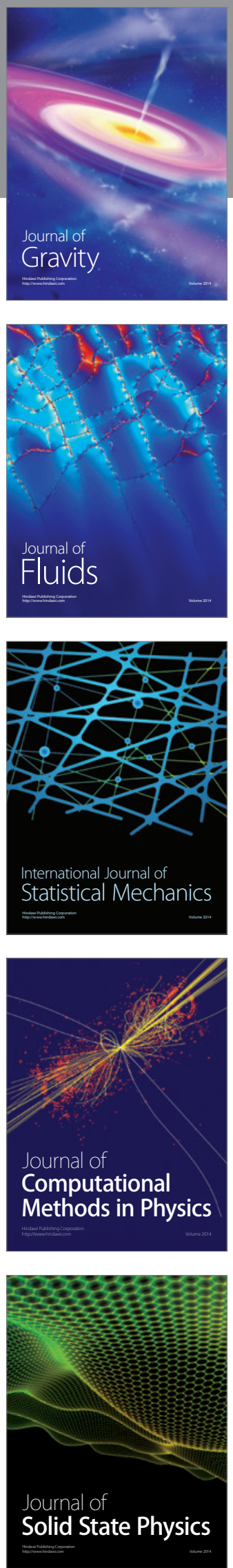

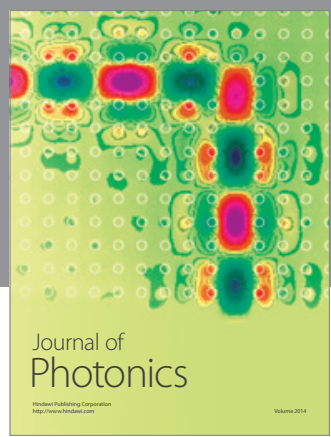

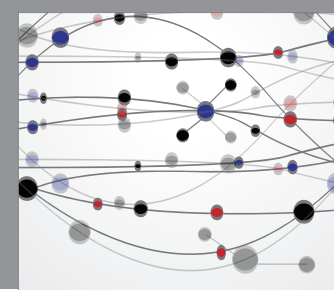

The Scientific World Journal

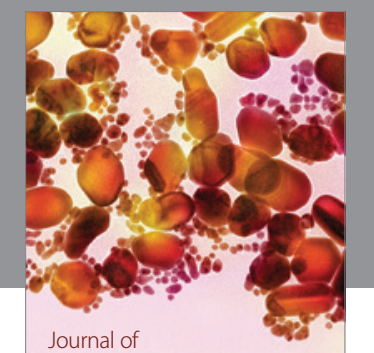

Soft Matter
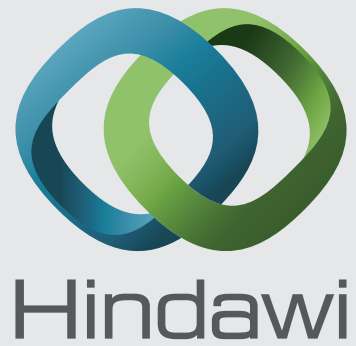

Submit your manuscripts at

http://www.hindawi.com
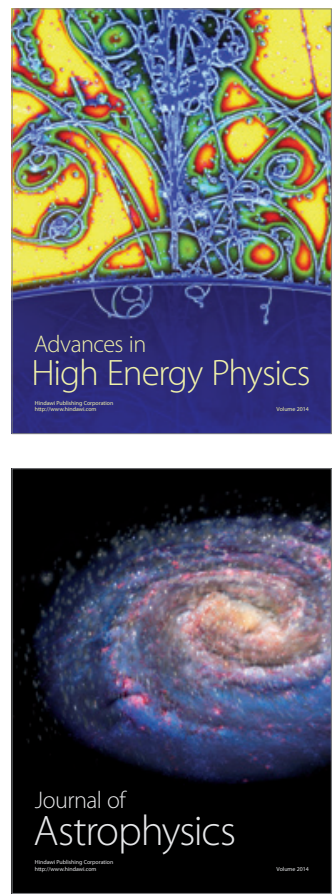
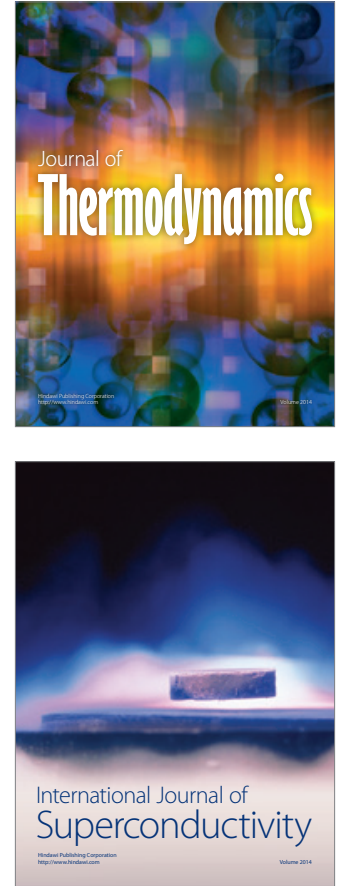
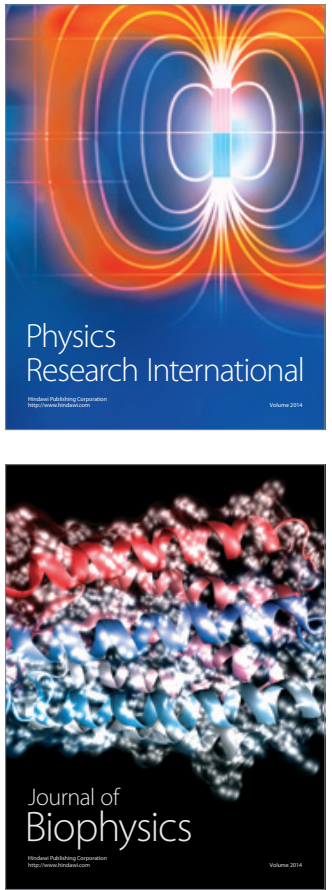
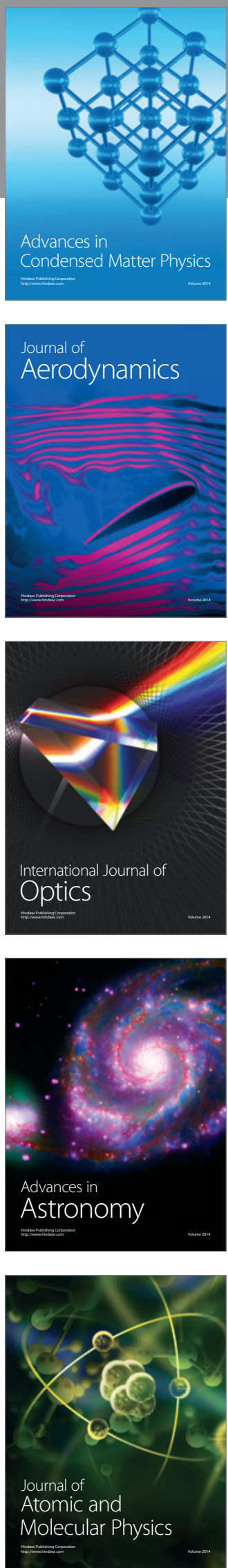